\title{
Medición Localizada de Contaminantes Atmosféricos y Variables Meteorológicas: Segunda Ley de la Termodinámica
}

Patricio R. Pacheco(1,3), Eduardo M. Mera(1) y Giovanni A. Salini(2)

(1) Depto. de Física, Facultad de Ciencias Naturales, Matemática y del Medio Ambiente, Univ. Tecnológica Metropolitana, Las Palmeras 3360, Santiago-Chile

(3) Universidad Tecnológica de Chile, Vicuña Mackenna 3864, Macul

(2) Depto. de Matemática y Física Aplicadas, Facultad de ingeniería, Universidad. Católica de la Santísima. Concepción, Alonso de Rivera 2850, Concepción-Chile

E-mail: patricio.pacheco@utem.cl; emera@utem.cl; gsalini@ucsc.cl; patrício.pacheco03@inacapmail.cl

Recibido Sep. 27, 2018; Aceptado Dic. 13, 2018; Versión final Ene. 20, 2019, Publicado Jun. 2019

\section{Resumen}

Desde la perspectiva de la teoría del caos, se analizan series de tiempo de contaminantes como material particulado fino y grueso y de monóxido de carbono junto a las variables meteorológicas, humedad relativa, velocidad del viento y temperatura. Las series surgen de mediciones en seis estaciones de monitoreo ubicadas en Santiago de Chile, de las cuales se seleccionaron dos, para un periodo de 3.25 años. Aplicando la segunda ley de la termodinámica, que es un principio general que impone restricciones a la dirección de la transferencia de calor, y a la eficiencia posible de la denominada máquina térmica (natural y artificial), se estudia la actividad antropogénica y su conexión con la dinámica meteorológica atmosférica. Las entropías de correlación permiten explicar esta conectividad, así como, el efecto de la proximidad geográfica en el proceso de difusión de los contaminantes.

\section{Localized Measurements of Atmospheric Pollutants and Meteorological Variables: The Second Law of Thermodynamics}

\begin{abstract}
Time series of pollutants such as fine and coarse particulate matter and carbon monoxide are analyzed from a chaos theory perspective, along with the meteorological variables, relative humidity, wind speed and temperature. The series arise from measurements in six monitoring stations located in Santiago, Chile, of which two were selected, for a period of 3.25 years. Applying the second law of thermodynamics, which is a general principle that imposes restrictions on the direction of heat transfer, and the possible efficiency of the so-called thermal machine (natural and artificial), anthropogenic activity is studied and its connection with the atmospheric meteorological dynamics. The correlation entropies allow explaining this connectivity as well as the effect of geographical proximity in the diffusion process of the pollutants.
\end{abstract}




\section{INTRODUCCION}

Todos los sistemas presentes en la naturaleza están sometidos a procesos irreversibles (Bénard, 1901; Brillouin, 1962; Haddad, 2017). Esto ocurre cuando un sistema y sus alrededores no pueden regresar a su estado inicial. Se observa que el fenómeno de la irreversibilidad según Prigogine (Prigogine, 1955) tiene carácter constructivo, destacando el "papel creativo del tiempo". Por lo tanto, supone -al menos a nivel macroscópico- una especie de anti-entropía: el universo del no equilibrio es un universo conexo. Esto nos revela que en los fenómenos de la naturaleza existe un dialogo profundo entre conectividad e irreversibilidad. Una magnitud que da cuenta de sistemas que tienen esas características es la entropía. Ésta es una variable fundamental que tiene diferentes definiciones y se mide de disímiles formas, según los distintos campos en que se aplica (Sinai, 1959; Dewar, 2005; Haddad, 2017).

Desde una perspectiva histórica, en física clásica la entropía de un sistema físico es proporcional a la cantidad de energía no disponible para hacer trabajo físico. En mecánica cuántica, von Neumann extendió la noción de entropía a sistemas cuánticos por medio de la matriz de densidad (Bengtsson y Życzkowski, 2017). En teoría de probabilidades, la entropía de una variable azarosa mide la incerteza respecto del valor que debe ser asumido por la variable (Parker, 2009). En teoría de información, la entropía de compresión de un mensaje (un archivo computacional) cuantifica la cantidad de información que es llevada por el mensaje en términos de la mejor razón de compresión sin perdidas (Shannon, 1948). En teoría de sistemas dinámicos, la entropía cuantifica la complejidad exponencial de los sistemas o el flujo promedio de información por unidad de tiempo (Sprott, 2006). En sociología, la entropía es el decaimiento natural de las estructuras (tales como leyes, organización, y convenciones) de un sistema social. Y en el sentido usual, la entropía significa desorden o caos. Al decir que la entropía es una medida del desorden se da a entender que la más alta entropía es el desorden más grande, lo que aparece cuando es considerada como una medida de la no disponibilidad de la energía de un sistema para hacer trabajo. Es un parámetro que representa el estado de desorden de un sistema atómico, iónico, o molecular. También es una medida del desorden en el universo o simplemente una medida de desorden en un sistema cualquiera (Ruelle, 2003; Liu et al., 2011).

La entropía es central en la Segunda Ley de la Termodinámica, la cual establece que en un sistema aislado cualquier actividad la incrementa. Esta función de estado es de punto; es decir, depende sólo de su estado inicial y final sin tener en cuenta la transición - o camino recorrido - para llegar desde el estado inicial al estado final de la transformación. Las funciones de estado aparecen en las tres leyes fundamentales de la termodinámica. En la Ley Cero de la Termodinámica comprende el concepto de temperatura, y en la Primera Ley, al de energía interna. Tanto la temperatura como la energía interna son funciones de estado que pueden emplearse para describir el estado termodinámico de un sistema. La entropía es una función de estado relacionada con la segunda ley de la termodinámica y, a una escala macroscópica, fue enunciada por primera vez por Rudolph Clausius en 1865. La Energía Térmica y el Trabajo, presentes en la Primera Ley, dependen del estado inicial, final e intermedio del sistema termodinámico, es decir dependen de la trayectoria entre los estados inicial y final. Sin embargo la Energía Interna depende solo del estado inicial y final del sistema y, además es una cantidad conservada. Para investigar la relación entre la entropía y la Segunda Ley de la Termodinámica, se consideraran los cambios de Entropía en procesos reales (irreversibles), mostrándose que la función Entropía depende solo del estado del sistema. Por lo tanto, el cambio de entropía cuando un sistema se mueve entre dos estados de equilibrio cualesquiera depende únicamente de los estados inicial y final.

En un ciclo reversible se define la entropía como:

$$
d S=\frac{\delta Q}{T}
$$

lo cual se interpreta afirmando que la variación elemental de entropía es igual al calor diferencial ( $\delta$ Q) transmitido a la temperatura T. Se representa en un diagrama T - S llamado "diagrama entrópico".

Es importante saber qué ocurre cuando hay varios focos térmicos (De Groot y Mazur, 1962; Moran y Shapiro, 2005). Si el sistema evoluciona variando su temperatura en varios pasos, a base de ponerse en contacto con distintos ambientes a diferentes temperaturas, intercambiará calor con cada uno de ellos, y ya no es posible hablar llanamente de calor de foco caliente $\left(Q_{c}\right)$ y calor de foco frío $\left(Q_{t}\right)$, sino que se está en presencia de una serie de calores $Q_{1}, Q_{2}, Q_{3}, \ldots$ que entran en el sistema desde focos a temperaturas $T_{1}, T_{2}, T_{3}, \ldots$. La desigualdad correspondiente al proceso, se conoce como desigualdad de Clausius:

$$
\frac{Q_{1}}{T_{1}}+\frac{Q_{2}}{T_{2}}+\frac{Q_{3}}{T_{3}}+\ldots \leq 0
$$


En notación técnica

$$
\sum_{i=1}^{n} \frac{Q_{T F i}}{T_{T F i}} \leq 0
$$

La variable TF es la temperatura asociada a cualquier fuente de calor. En el límite cuando $n$ tiende a infinito la sumatoria en ec. (3) se transforma en una integral (en el sentido de Riemann), por lo que la desigualdad de Clausius se escribe para un proceso continuo como:

$$
\int \frac{d Q}{T} \leq 0
$$

donde la igualdad corresponde, ahora, a ciclos reversibles y la desigualdad a irreversibles.

Si se supone un ciclo irreversible formado por un camino irreversible (C) que lleva del estado 1 al 2 y vuelve por un camino reversible $\left(C^{\prime}\right)$, en este caso se tiene la desigualdad

$$
c \int_{1}^{2} \frac{d Q_{1}}{T}+c \int_{2}^{1} \frac{d Q_{R}}{T}<0
$$

que equivale a decir que la integral por el camino reversible es mayor que por el irreversible:

$$
-c \int_{2}^{1} \frac{d Q_{R}}{T}>c \int_{1}^{2} \frac{d Q_{1}}{T}
$$

Pero la integral por el camino reversible es justamente la diferencia entre la entropía inicial y la final. Por tanto

$$
\Delta S=-\left(S_{1}-S_{2}\right)>c \int_{1}^{2} \frac{d Q_{1}}{T}
$$

Se puede transformar esta desigualdad en una igualdad introduciendo la producción de entropía $\sigma_{g e n}$ :

$$
\Delta \mathrm{S}=\mathrm{S}_{2}-\mathrm{S}_{1}=\mathrm{c} \int_{1}^{2} \frac{\mathrm{dQ}_{\mathrm{l}}}{\mathrm{T}}+\sigma_{\mathrm{gen}}
$$

$\sigma$ mide el efecto de las irreversibilidades presentes en el interior del sistema durante el proceso, su valor dependerá de la naturaleza del proceso y no solo de los estados inicial y final. Por tanto no es una propiedad. Al aplicar el balance de entropía a un sistema cerrado es esencial considerar los requisitos impuestos por el segundo principio a la generación de entropía: este principio requiere que la generación de entropía tenga un valor positivo a nulo, es decir, $\sigma>0$, proceso internamente irreversible, $\sigma=0$, proceso internamente reversible (Moran y Shapiro, 2005). Así, la desigualdad de Clausius equivale a decir que la producción de entropía en un sistema es siempre positiva (en un proceso irreversible) o nula (en uno reversible) pero nunca negativa.

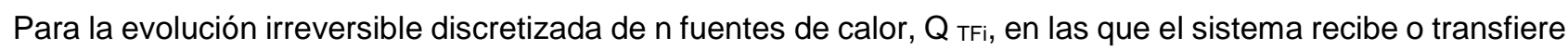
calor al sistema y llamando $T_{\mathrm{FTi}}$ a la temperatura asociada a la $i$ - ésima fuente, la ecuación de Clausius queda:

$$
\mathrm{dS}=\delta \sigma_{\mathrm{p}}+\sum_{\mathrm{i}=1}^{\mathrm{n}} \frac{\delta \mathrm{Q}_{\mathrm{T} F_{\mathrm{i}}}}{\mathrm{T}_{\mathrm{T} F_{\mathrm{i}}}}
$$

En el caso de darse un proceso reversible y adiabático, según la ecuación (9), d $S=0$. Vale decir, el valor de la entropía es constante (proceso isentrópico).

¿Es posible confirmar cuantitativamente las restricciones de la Segunda Ley al balance y flujo de entropía, utilizando una base de datos empírica de Series de Tiempo de Variables Meteorológicas y de Concentración de contaminantes atmosféricos localizados referida a procesos irreversibles (Audelo-Vucovich et al., 2015)? 
La gran dificultad estriba en calcular la entropía en Series de Tiempo. No existen muchos trabajos que traten de conciliar, en primera aproximación, el modelo clásico de la segunda ley de la termodinámica y su relación con la entropía de bases de datos disponibles de medio ambiente, precisamente por la dificultad de enfrentar la función de estado denominada entropía.

\section{MATERIALES Y MÉTODOS}

La verificación experimental de la hipótesis del presente artículo, que inicialmente consideró 6 estaciones que entregaron 1024704 datos, se rebajó a dos localidades (Puente Alto y La Florida, dos comunas de Santiago de Chile) porque eran las que tenían menos datos faltantes; el objetivo de esta decisión fue la de ser lo menos invasivo posible respecto de la data, buscando satisfacer criterios de confiabilidad y validez. Los datos fueron analizados por dos Softwares: Golden Surfer y CDA. Este último software produce diversos parámetros de interés en esta investigación, siendo el coeficiente de Lyapunov $(\lambda)$, la dimensión de Correlación, el exponente de Hurst $(\mathrm{H})$ y la Entropía de Correlación los más relevantes dado su conectividad con principios termodinámicos generales. A continuación se presenta el detalle del procedimiento seguido.

\section{Área de estudio}

La ciudad de Santiago de Chile, se sitúa a $33.5^{\circ}$ Sur y $70.8^{\circ}$ Oeste. Posee una población de alrededor de 6 millones de habitantes, el $40 \%$ de la población del país, en una superficie de alrededor de $135 \mathrm{Km}^{2}$. Está ubicada en el centro del país, a una altura de $520 \mathrm{msnm}$, aproximadamente. La altitud por sobre el nivel del mar crece de Poniente a Oriente. Está rodeada por dos sistemas montañosos: las cordilleras de Los Andes y de La Costa.

\section{Los datos y tratamiento de datos}

Las series de tiempo de concentraciones horarios de contaminantes atmosféricos y de variables meteorológicas fueron recopiladas desde la Red MACAM III (Red de monitoreo de calidad del aire para la Región Metropolitana de Santiago de Chile) que aporta mediciones al Sistema SINCA (Sistema de Información Nacional de Calidad del Aire) y dependientes del MMA (Ministerio del Medio Ambiente de Chile), para un periodo de tiempo desde el año 2010 hasta el año 2013, correspondiendo a 39 meses o a 3,25 años, con un registro total de 341568 datos. Las variables de contaminación fueron $\mathrm{PM}_{10}$ (material particulado de 10 micrómetros de diámetro), $\mathrm{PM}_{2.5}$ (material particulado de 2.5 micrómetros de diámetro) y CO (Monóxido de Carbono). A su vez, las variables meteorológicas fueron temperatura ( $T$ ), humedad relativa (HR) y velocidad del viento (VV). Las estaciones de monitoreo consideradas fueron seis: La Florida (EML), Las Condes (EMM), Santiago-Parque O'Higgins (EMN), Pudahuel (EMO), Puente Alto, (EMS) y Quilicura (EMV) (Fig. 1). Pero el estudio, por la razones indicadas, las restringió a dos: EMS y EML. Para el llenado de Series de Tiempo incompletas se aplicó el software Golden Surfer (Ramos et al., 2016) y, dentro de las técnicas de interpolación que propone, la de Kriging para datos faltantes (este método pondera los valores medidos circundantes para calcular una predicción de una ubicación sin mediciones, el motivo de su aplicación es que es funcional para efectos comparativos respecto de estaciones de monitoreo próximos vecinos). Para el análisis no lineal de las series de tiempo se utilizó el Software CDA, Chaos Data Analyzer (Sprott, 2006) (aplica elementos de la Teoría del Caos para el análisis de Series de Tiempo).

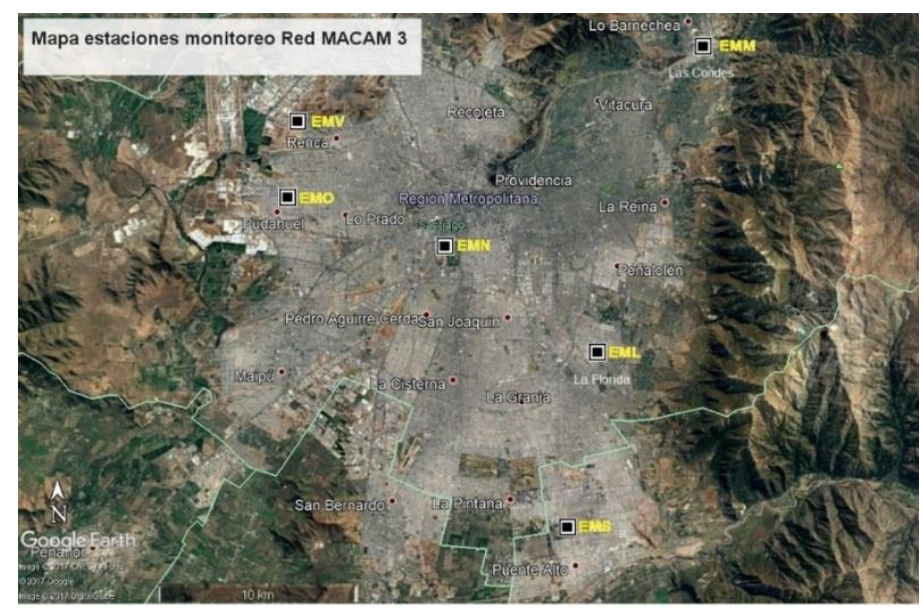

Fig. 1: Plano de algunas estaciones de monitoreo del estudio para la ciudad de Santiago de Chile. 
En la figura 2 se muestra las Series de Tiempo de la velocidad del viento, en estaciones de monitoreo en estudio (EML y EMS). Por su parte, en la figura 3 se presenta un diagrama esquemático con parámetros de interés en este estudio, para ambas estaciones de monitoreo (EML y EMS).

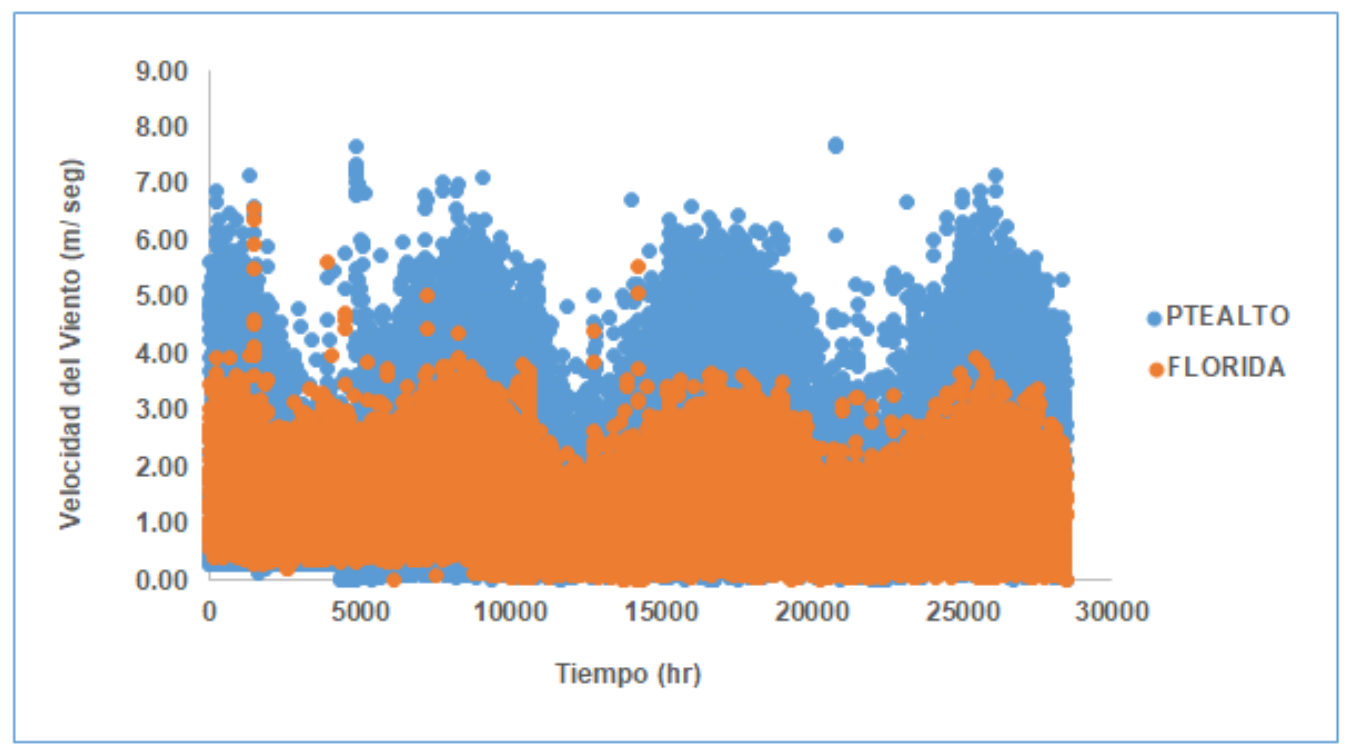

Fig. 2: Evolución del régimen de magnitudes de la velocidad del viento (VV) con el tiempo, en las estaciones de monitoreo de Puente Alto (EMS) y La Florida (EML).

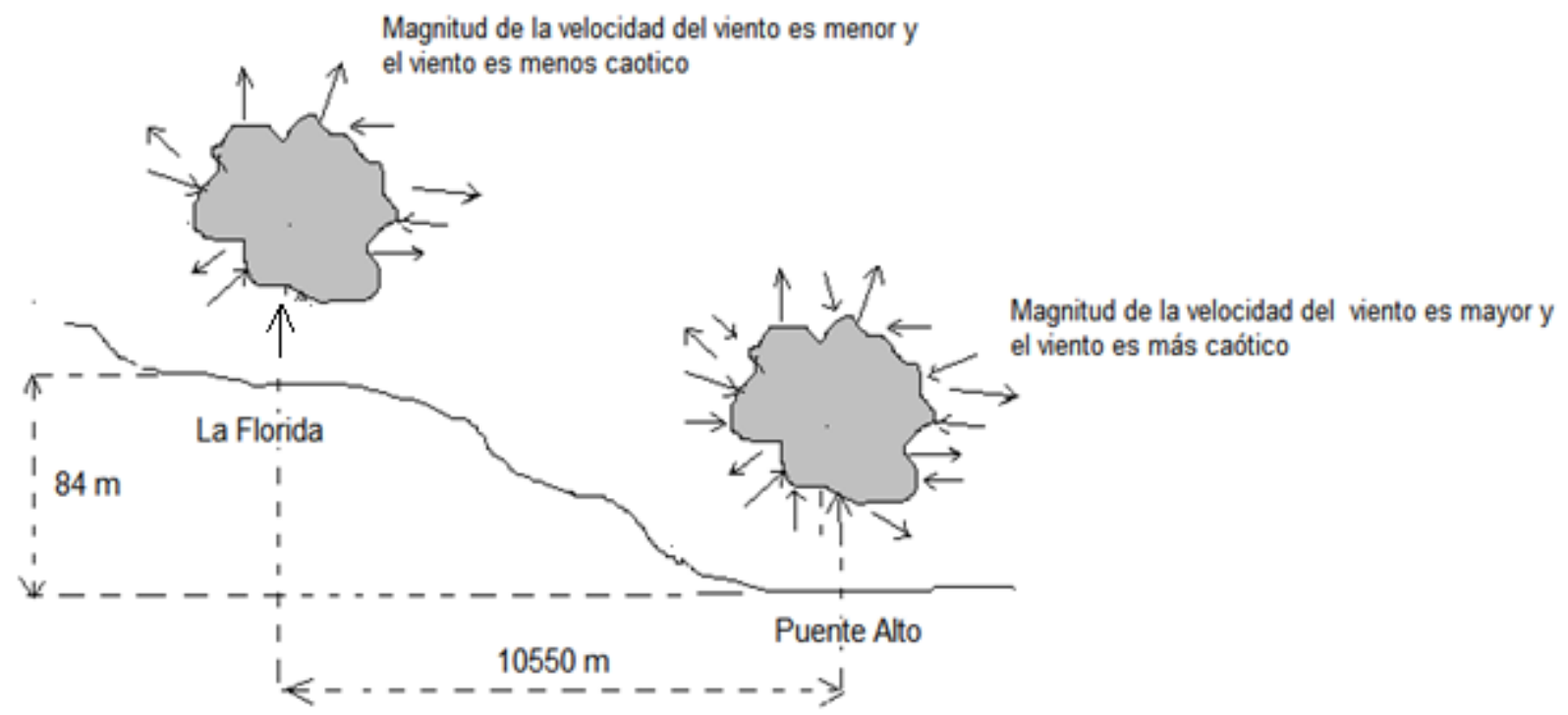

Fig. 3: Esquema de representación seccional para EML y EMS considerando sus regímenes de vientos, elevación y distancia de separación (ver Tabla 1 y 2 , columna de coeficientes de Lyapunov $\lambda, \lambda_{\mathrm{EMS}}>\lambda_{\mathrm{EML}}$ )

\section{Cálculos de coeficientes}

Existen diferentes herramientas de cálculo matemático (como el Software Chaos Data Analyzer -CDA(Sprott, 2006), que aplicado a series de tiempo -sintéticas o empíricas-permite obtener los coeficientes de Lyapunov, que confirman o no la naturaleza caótica de una serie temporal asociado a los contaminantes y variables meteorológicas. Por su parte, el exponente de Hurst nos da la persistencia. A su vez, se obtienen la dimensión de correlación y la entropía de Kolmogorov (Kolmogorov, 1983). Este último valor representa un flujo de entropía en bits/unidades de tiempo, información que permite identificar el tipo de flujo (negativo, positivo o cero). Si es reversible o irreversible y, por extensión, su dependencia de la trayectoria (o el camino recorrido en el proceso). El número de eigenvalores de la matriz de correlación, tal como la dimensión de correlación, es una medida de la complejidad del sistema (Sprott, 2006). Para valores de dimensión de correlación mayores a 5, los datos son esencialmente aleatorios. Obtener la complejidad del sistema (C) (Sprott, 2006; López-Corona et al, 2015) y su pérdida de información es un indicador de su predictibilidad ( $\mathrm{C}=$ 0) y no predictibilidad ( $>$ 0), y de su sustentabilidad (Wackernagel et al., 2002; Sayre, 2008). 
Siguiendo a Farmer y colaboradores (Farmer et al., 1983), se tiene que una de las diferencias esenciales entre el comportamiento caótico y el predecible es que las trayectorias caóticas generan continuamente nueva información mientras que las trayectorias predecibles no lo hacen. La entropía métrica hace esta noción más rigurosa. Además de proporcionar una buena definición de "caos", la entropía métrica proporciona una forma cuantitativa para describir "cuan caótico" es un sistema dinámico. Sea una trayectoria $x(t)=\left[x_{1}(t), x_{2}(t), \ldots\right.$, $\left.\mathrm{x}_{\mathrm{n}}(\mathrm{t})\right]$, en el espacio de fases de dimensión $d$ de un sistema dinámico, según la figura 4:

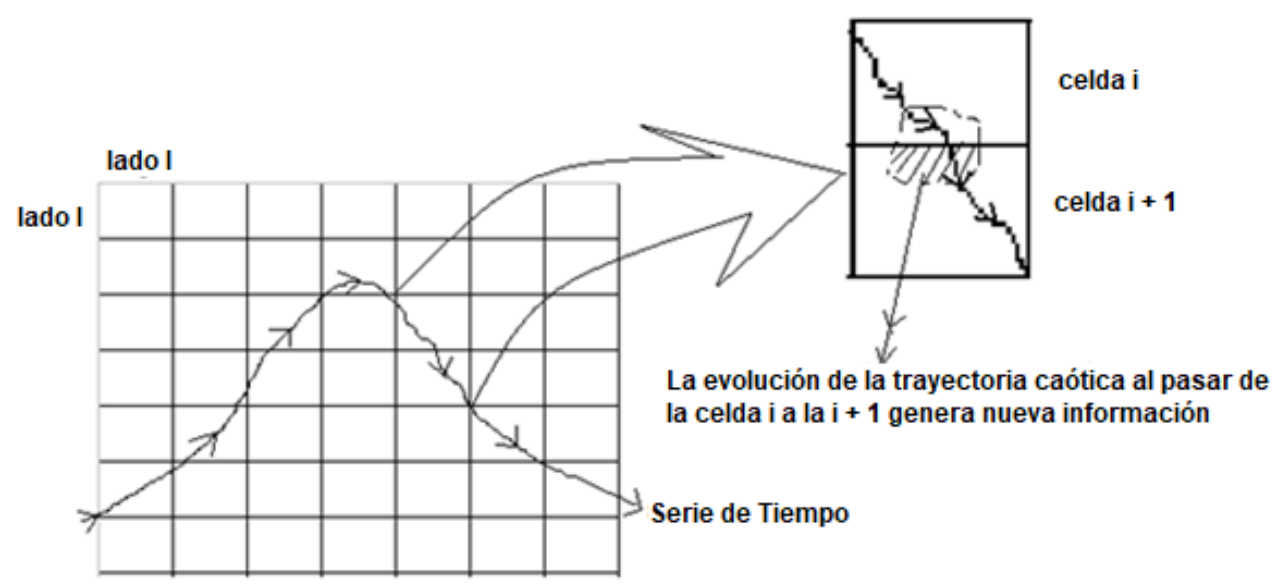

Fig. 4: Diagrama esquemático mostrando la evolución temporal de trayectoria caótica.

Si se supone que este espacio está dividido en celdas de tamaño $I^{d}$ siendo d la dimensión de ese espacio, y, además, que se mide el estado del sistema a intervalos de tiempo $\tau$ uniformemente separados. Sea $P_{0} \ldots$ la probabilidad conjunta de que en el instante $t=0$ el sistema se encuentre en la caja $i$, en $t=\tau$ en $i 1 \ldots$ y en n t en in. Según la teoría de Shannon, la entropía de Kolmogorov, K, se define como

$$
K=-\sum_{0 \ldots . n} P_{0 \ldots n} \log P_{0 \ldots n}
$$

Vale decir, es proporcional a la información requerida para localizar el sistema en una trayectoria especial que recorra las celdas i $0 . . .$. i n con precisión determinada. Entonces, la entropía de Kolmogorov (Kolmogorov, 1983) es el promedio de pérdida de información (Shannon, 1948; Brillouin, 1962) cuando "P" (lado de la celda en unidades de información) y т (tiempo) se hacen infinitesimales (Martínez y Vinagre, 2016):

$$
S_{K}=-\lim _{T \rightarrow 0} \lim _{1 \rightarrow 0} \lim _{n \rightarrow \infty} \frac{1}{n T} \sum_{0 \ldots . n} P_{0 \ldots . . .} \log P_{0 \ldots . . n}
$$

Esta entropía se expresa en bits de información/seg o bits/órbitas para un sistema continuo y bits/iteración para un sistema discreto (Shaw, 1981; Cohen y Procaccia, 1985).

(Nota: orden de prioridad en el cálculo de límites:

$1^{\circ}$. $\mathrm{n} \rightarrow \infty, 2^{\circ}$. $\mathrm{I} \rightarrow 0$, para eliminar la dependencia de la partición elegida. $3^{\circ}$. $\tau \rightarrow 0$ sólo es necesario en sistemas continuos).

La diferencia de entropía de Kolmogorov $\left(\Delta S_{K}\right)$ entre una celda y otra $\left(S_{K n+1}-S_{K}\right)$ representa la información adicional que se necesita para saber en qué celda $\left(i_{n+1}\right)$ se va a encontrar el sistema en el futuro. Por lo tanto, esta diferencia mide la pérdida de información del sistema en el tiempo.

En resumen, para el cálculo de la entropía de Kolmogorov (Martínez y Vinagre, 2016) se procede de la siguiente manera: en primer lugar, se comprueba si la entropía se encuentra entre cero e infinito $\left(0<S_{k}<\infty\right)$, lo cual permite verificar la presencia de un comportamiento caótico. Si la entropía de Kolmogorov es igual a 0 , no se pierde información y el sistema es regular y predecible. Si Sk es infinito, el sistema es totalmente aleatorio y es imposible hacer cualquier predicción. En segundo lugar, se determina la cantidad de información que se necesita para predecir el comportamiento futuro de un sistema; en este caso, un sistema interactivo contaminante-variable atmosférica. En tercer lugar, se calcula la velocidad con la que el sistema pierde (o desactualiza) información en el tiempo. Finalmente, establece el horizonte máximo de predictibilidad del sistema, aquella frontera a partir de la cual no se puede realizar predicción alguna, ni elaboración de escenarios (Salini y Pérez, 2015). 


\section{RESULTADOS}

En las tablas 1 y 2 se presentan los parámetros no lineales de salida para las Series de Tiempo de las estaciones de monitoreo EML y EMS (los datos de entrada son las Series de Tiempo (cada una con 28464 pares ordenados), la dimensión de incrustamiento, $\mathrm{D}=3$ y el número de intervalos de muestra sobre los cuales cada par de puntos es seguido antes de que un nuevo par sea seleccionado, $n$, el programa asigna un valor por default pero puede ser modificada). Por su parte, en la tabla 3 se muestra el cálculo para la entropía de contaminantes y de variables meteorológicas consideradas en este estudio, para las mismas estaciones de monitoreo.

Tabla 1: Cuadro resumen parámetros caóticos, comuna de Puente Alto (EMS)

\begin{tabular}{|l|c|c|c|c|}
\hline \multicolumn{1}{|c|}{ Variable } & $\lambda$ & $H$ & Dimensión de Correlación & Entropía \\
\hline PM2.5 & $0.542 \pm 0.010$ & 0.973 & $4.012 \pm 0.213$ & 0.531 \\
\hline PM10 & $0.421 \pm 0.010$ & 0.969 & $4.133 \pm 0.140$ & 0.452 \\
\hline CO & $0.439 \pm 0.014$ & 0.953 & $4.686 \pm 0.470$ & 0.394 \\
\hline TEMP & $0.489 \pm 0.011$ & 0.990 & $3.171 \pm 0.413$ & 0.395 \\
\hline HR & $0.725 \pm 0.011$ & 0.992 & $3.697 \pm 0.192$ & 0.416 \\
\hline VV & $0.880 \pm 0.010$ & 0.957 & $4.250 \pm 0.045$ & 0.478 \\
\hline
\end{tabular}

Tabla 2: Cuadro resumen parámetros caóticos, comuna de La Florida (EML)

\begin{tabular}{|l|c|c|c|c|}
\hline \multicolumn{1}{|c|}{ Variable } & \multicolumn{1}{c|}{$\lambda$} & $H$ & Dimensión de Correlación & Entropía \\
\hline PM2.5 & $0.610 \pm 0.010$ & 0.973 & $4.179 \pm 0.148$ & 0.460 \\
\hline PM10 & $0.489 \pm 0.011$ & 0.967 & $4.141 \pm 0.145$ & 0.520 \\
\hline CO & $0.508 \pm 0.014$ & 0.959 & $4.104 \pm .0 .566$ & 0.557 \\
\hline TEMP & $0.472 \pm 0.012$ & 0.989 & $2.681 \pm 0.234$ & 0.409 \\
\hline HR & $0.631 \pm 0.011$ & 0.991 & $3.310 \pm 0.367$ & 0.425 \\
\hline VV & $0.772 \pm 0.009$ & 0.976 & $4.465 \pm 0.088$ & 0.496 \\
\hline
\end{tabular}

Tabla 3: Cuadro resumen de Entropías de Contaminantes y de Variables Meteorológicas (ambas estaciones)

\begin{tabular}{|l|c|c|c|c|}
\hline \multicolumn{1}{|c|}{ Localidad } & Clave & MSNM & Entropía Contaminantes & Entropía Variables Meteorológicas \\
\hline Puente Alto & EMS & 700 & 1.377 & 1.289 \\
\hline La Florida & EML & 784 & 1.537 & 1.33 \\
\hline
\end{tabular}

\section{ANÁLISIS}

De la Tabla 3 se desprende que el flujo de entropía (en bits/hora) satisface las siguientes condiciones:

$$
\begin{aligned}
& \Delta S \text { K VARIABLES METEOROLOGICOS }=S_{k} \text { EMS }-S_{k} \text { EML }<0 \\
& \Delta S_{k} \text { CONTAMINANTES }=S \text { K EMS }-S_{k} \text { EML }<0
\end{aligned}
$$

El índice de calidad del aire, promedio, para la estación EMS es 69 y para la estación EML es del orden de 74 (Índice de calidad del aire, 2018), ambos calificados como moderados. Estas cifras pueden inducir la autocomplacencia o autoengaño, pues desde el punto de vista de la termodinámica y de la entropía, existe un flujo de material contaminante, al que, además, contribuye la entropía de las variables meteorológicas, desde estación EML a estación EMS.

El análisis caótico de las series de tiempo asume el carácter irreversible de los procesos termodinámicos que representan. Esto significa que, según las localidades EML y EMS, el valor obtenido de la entropía de correlación, entrega la ganancia de desorden por adquisición de nueva información debida a las relaciones entre dos o más variables en la zona de obtención de la serie de tiempo. El sistema caótico continuamente gana más información, volviéndose más complejo, lo que afecta su horizonte de predictibilidad (Salini y Pérez, 2015). Al demostrar que los datos medidos representan procesos caóticos, significa que estos procesos consumen considerable energía e información. Como la entropía de los contaminantes es mayor son los más complejos (Sprott, 2006), a la vez que son muy sensibles a las condiciones iniciales (que pueden ser fluctuaciones ambientales inducidas o no inducidas), esta es una característica de un régimen caótico. En la Tabla 3 se observa que la columna de los contaminantes es la de mayor entropía lo que explica, en parte, su conectividad y sostenibilidad en el tiempo, por intercambio de energía con la atmósfera tensionándola, en 
desmedro de ésta, lo que puede, dentro de muchas razones, explicar el origen de eventos tales como variaciones climáticas inusuales, alerta, pre-emergencia o emergencia por contaminación. Junto a la acción antropogénica revelan el cuadro de detrimento general atmosférico que se observa en la zona.

Los flujos entrópicos tienen un comportamiento muy dinámico, irreversible y caótico (fig. 5), pues su existencia se manifestará por la sola irrupción de las diferencias de $\Delta S$. Así para cada emisor de contaminantes y su entorno vecino, surgida la diferencia, se producirá flujo en el tiempo de entropía que puede ser de tipo neguentrópico:

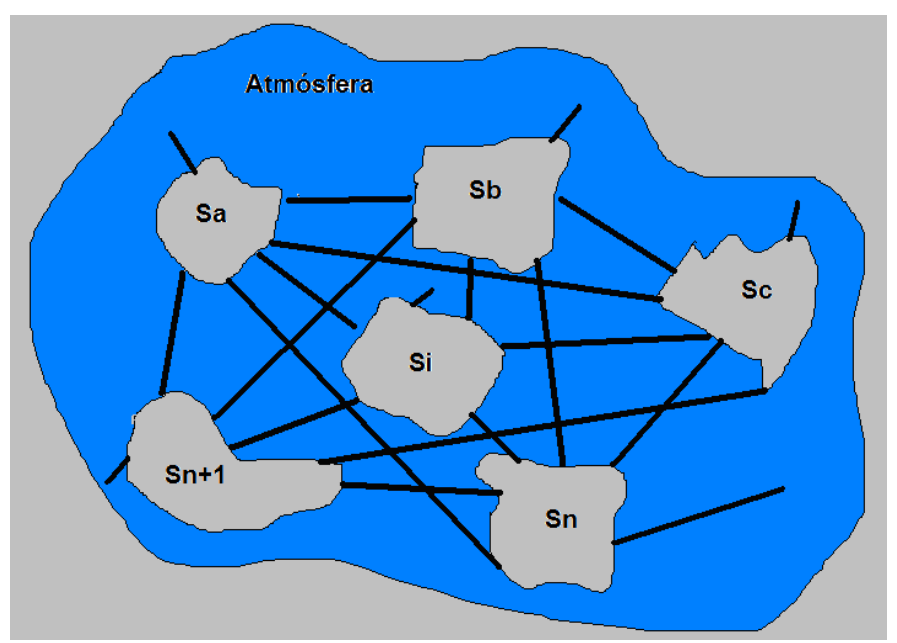

Fig. 5: Los flujos entrópicos son altamente dinámicos y se originan ante cualquier $\Delta S \neq 0$.

Si un sistema aislado no está en equilibrio, evolucionará naturalmente en la dirección en la cual su entropía aumenta, ya que éstos son los procesos que conducen al sistema hacia el estado de máxima probabilidad o de equilibrio estadístico. Así, los procesos que son más probable que ocurran en un sistema aislado son aquellos para los cuales (López - Corona, et al., 2015):

$d S \geq 0$

La desigualdad es válida cuando el sistema no está inicialmente en equilibrio y los procesos son irreversibles. El estudio que se presenta responde a las características de un sistema que no está aislado, su entropía puede disminuir debido a la interacción con otros sistemas, cuyas entropías deben cambiar también. Pero la cantidad total de cambios de entropía de todos los sistemas involucrados en el proceso debe estar de acuerdo con la ecuación (14), siendo d $S=0$ para un proceso reversible y d $S>0$ para uno irreversible cuando el sistema no está inicialmente en equilibrio.

La entropía de correlación se mide en bits/hora. Así, para la situación que se estudia:

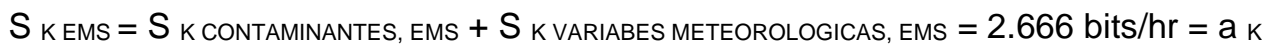

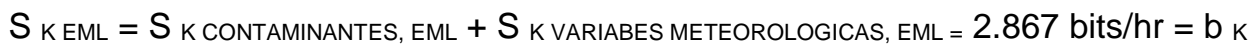

A partir de esto, la entropía termodinámica horaria para procesos irreversibles del estudio vale aproximadamente $\mathrm{S}_{\mathrm{T}, \mathrm{EMS}} \approx \mathrm{a} \approx 2.7$ bits y $\mathrm{S}_{\mathrm{T}, \mathrm{EML}} \approx \mathrm{b} \approx 2.9$ bits. Así, usando la definición de entropía termodinámica, la diferencia será:

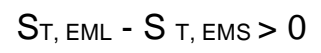

satisfaciendo la condición de proceso irreversible. Por su parte, esto se sigue del siguiente argumento. Se puede observar que según la ecuación (8), para proceso irreversible:

$$
\sigma_{\text {gen }}^{\text {LOCALIZACION }}=\left(S_{2}-S_{1}\right)-c \int_{1}^{2} \frac{d Q_{1}}{T}>0
$$

Para el caso en estudio, obtenemos:

$$
\sigma_{\text {gen }}^{\text {EMS }}=\left(S_{2}-S_{1}\right)^{\text {EMS }}-a^{\text {EMS }}>0 \Rightarrow\left(S_{2}-S_{1}\right)^{\text {EMS }}>a^{\text {EMS }} \forall a \in R
$$




$$
\sigma_{\text {gen }}^{\mathrm{EML}}=\left(\mathrm{S}_{2}-\mathrm{S}_{1}\right)^{\mathrm{EML}}-\mathrm{b}^{\mathrm{EM}}>0 \Rightarrow\left(\mathrm{S}_{2}-\mathrm{S}_{1}\right)^{\mathrm{EML}}>\mathrm{b}^{\mathrm{EML}} \forall \mathrm{b} \in \mathrm{R}
$$

De estas dos ecuaciones se desprende que:

$$
\begin{aligned}
& \sigma_{\text {gen }}^{\mathrm{EM}}-\sigma_{\mathrm{gen}}^{\mathrm{EMS}}=\left(\mathrm{S}_{2}-\mathrm{S}_{1}\right)^{\mathrm{EML}}-\left(\mathrm{S}_{2}-\mathrm{S}_{1}\right)^{\mathrm{EMS}}+a^{\mathrm{EMS}}-\mathrm{b}^{\mathrm{EML}} \\
& \sigma_{\mathrm{gen}}^{\mathrm{EM}}-\sigma_{\mathrm{gen}}^{\mathrm{EMS}}=\left(\mathrm{S}_{2}-\mathrm{S}_{1}\right)^{\mathrm{EML}}-\left(\mathrm{S}_{2}-\mathrm{S}_{1}\right)^{\mathrm{EMS}}-0.2
\end{aligned}
$$

La expresión $\left(\mathrm{S}_{2}-\mathrm{S}_{1}\right)$ EML sostiene el proceso de generación de entropía, lo que es consecuente con el mayor valor de entropía de EML para proceso irreversible (Tabla 3). Finalmente, la diferencia $\left(S_{2}-S_{1}\right)$ sólo depende de los estados final e inicial y no de la trayectoria seguida para conectar ambos estados (tal como señala el miembro izquierdo de la ecuación (6) y de la ecuación (7)).

A partir del tratamiento caótico de los datos experimentales de contaminantes y de variables meteorológicas referidos a dos localidades se verifica, en primera aproximación, la Segunda Ley de la Termodinámica. Depende de la diferencia entre los estados entrópicos que caracterizan a las estaciones EML y EMS, con independencia del camino seguido para conectarlos. Así, el efecto de la distancia de separación entre ambas localidades es despreciable (Mahulikar y Herwig, 2009).

\section{DISCUSION}

Para medidas mitigadoras, existe una gran desigualdad en el Gran Santiago en cuanto a acceso a áreas verdes (generación de micro meteorología relacionada directamente con las variables meteorológicas), que contribuyen a aminorar este problema (Informe Min. de Viv. y Urbanismo, 2018). A modo de muestra, Puente Alto (EMS) $(1,5)$, El Bosque $(1,9)$ y Cerro Navia $(2,1)$ son las comunas que presentan menor cantidad de áreas verdes accesibles por habitante, mientras que Vitacura $(7,4)$, Independencia $(5,9)$ y Las Condes $(5,6)$, son las que tienen más.

La zona noreste (Lo Barnechea) y la zona sur (Puente Alto (EMS), La Pintana, La Florida (EML), entre otros) presentan la peor accesibilidad. Las comunas con menos áreas verdes son principalmente las periféricas debido principalmente a un tema económico. Si bien Puente Alto presenta una accesibilidad de $1,5 \mathrm{~m}^{2} / \mathrm{ha}$, hay barrios dentro de dicha comuna que tienen buena accesibilidad (sobre los $3 \mathrm{~m}^{2} / \mathrm{hab}$ ), como lo son la Villa Porvenir, Plaza de Puente Alto, Sector Ejército Libertador y Sector Regimiento. Sin embargo, existen zonas como la noreste (El Peral) y sureste (Las Vizcachas) que están críticas en áreas verdes, incluso más bajos que el promedio comunal. Las autoridades tienen que actuar gestionando suelos para tener nuevas áreas verdes, dado que Santiago tiene poco suelo vacante disponible para esta función social. Las áreas verdes, en general, generan tres efectos en la población. Desde el punto de vista del interés de este artículo, regulan la temperatura ambiente lo que incide en los flujos térmicos y en la entropía de las variables meteorológicas locales, reducen la contaminación acústica, etc. En el aspecto social estimulan espacios de interacción y mixticidad social, lo cual es importante en una ciudad segregada como Santiago, aportando a la salud de las personas al disminuir los niveles de ansiedad e incluso depresión en la población. Finalmente cuando las áreas verdes son de buena calidad, buen diseño, transporte, pueden aumentar las plusvalías de las viviendas hasta en un $50 \%$. Este triple valor justifica que sea un derecho social. Falta una política macro que priorice a las personas por sobre la explotación comercial como lo hacen las inmobiliarias. Quizás en esos intereses este entrampada el establecer una nueva ley.

Cabe destacar que se consideraron áreas verdes que están dentro del radio urbano de la ciudad, accesibles al público, que tienen más de 500 metros cuadrados, gratuitas o con entrada accesible y que estuvieran en buenas condiciones y consolidadas. Quedaron fuera las áreas naturales o semi-naturales, bandejones de autopistas, cementerios, campos de golf, Cerro Renca, Bosque Panul o sitios eriazos (Informe Ministerio de Vivienda y Urbanismo, 2013).

\section{CONCLUSIONES}

Este estudio, de primera aproximación, muestra la relación entre la Segunda Ley de la Termodinámica y la entropía. Se utiliza un conjunto de datos reales, en la forma de Series de tiempo, de un sistema de Variables Meteorológicas - Contaminantes Atmosféricos localizadas, en las que se verifica su caoticidad $(\lambda>0,5<\mathrm{H}$ $<1$, Dimensión de Correlación $<5$ ) y se les calcula la entropía $\left(S_{k}\right)$. Esto permite mostrar que los flujos de entropía: (i) se originan por las diferencias entre la Entropía en EMS (mínimo desorden) - Entropía EML 
(máximo desorden) y (ii) no dependen de la variable de longitud (horizontal y vertical) para distancias pequeñas ya que dependen de las diferencias de la función de estado entropía.

El cambio de entropía es el mismo para todos los procesos que ocurren entre un conjunto de estados inicial y final. Si no fuera así, se violaría la Segunda Ley de la Termodinámica. Como consecuencia de la aplicación de esta Ley, la variación en el tiempo de la entropía de los contaminantes y de las variables meteorológicas es de tipo neguentrópico (de EML(fuente) a EMS (sumidero)). Esta característica es la que lo hace comparable, en su comportamiento, a un sistema aislado (el cambio en la Entropía total del sistema aumenta). Cuando un sistema pasa de un estado a otro la variación de su entropía es independiente de los procesos realizados, ya que este cambio está determinado por las probabilidades de las particiones inicial y final que lo conduzcan hacia la máxima probabilidad o al equilibrio térmico.

\section{AGRADECIMIENTOS}

Los autores agradecen el financiamiento parcial de este trabajo a la Dirección de Investigación y Desarrollo Académico de la Universidad Tecnológica Metropolitana, a la Dirección de Investigación e Innovación y a la Facultad de Ingeniería de la Universidad Católica de la Santísima Concepción.

\section{REFERENCIAS}

Audelo-Vucovich. E., C. Vázquez-Cruz y F., Beristain, Tendencia de la Dinámica No-Lineal en una pre contingencia Ambiental causada por Partículas en Suspensión, doi: 10.4067/S0718-07642015000400004, Información Tecnológica, 26(4), 21-28 (2015)

Bénard, H., Les tourbillons cellulaires dans une nappe liquide propageant de la chaleur par convection en régime permanent, Ann. Chim. Phys, 23, 62-164 (1901)

Bengtsson, I. y K. Życzkowski, Geometry of Quantum States: An Introduction to Quantum Entanglement, 301, $2^{\text {nd }}$ Ed., Cambridge University Press, Cambridge, England (2017)

Brillouin, L., Science and Information Theory, $2^{\text {nd }}$ Ed., Academic Press Inc, New York, USA (1962)

Chen, Y., J. Wang y J. Feng, Understanding the Fractal Dimensions of Urban Forms through Spatial Entropy, doi: 10.3390/e19110600, Entropy, 19(11), 600, 1-18 (2017)

Cohen, A. e I. Procaccia, Computing the Kolmogorov entropy from time signals of dissipative and conservative dynamical systems, Physical Review A, 31, 1872-1882 (1985)

De Groot, S. R y P. Mazur, Non - equilibrium Thermodynamics, North - Holland: Amsterdam, Netherlands (1962), reprinted by Dover, London, (1985).

Dewar, R. C., Maximun entropy production and the fluctuation theorem, J. Phys. A. Math. Gen., 38, 1371-1381 (2005)

Farmer J.D., E. Ott y J. A. Yorke, The Dimension of Chaotic Attractors, Physica D, 9, 153-180 (1983)

Geweke, J. y S. Porter-Hudak, The estimation and application of long memory time series models, doi: 10.1111/j.14679892. 1983.tb00371.x, Journal of Time Series Analysis, 4, 221-238(1983)

Haddad, W., Thermodynamics: The Unique Universal Science, doi:10.3390/e19110621, Entropy, 19(11), 621(2017)

Hurst, H.E., R.P. Black e Y.M. Simaika, Long-term storage: an experimental study, $1^{\text {st }}$ Ed., Constable, London, UK (1965) Índice de Calidad del Aire, Promedio, para la estación EMS y EML (2018)

Informe Ministerio de Vivienda y Urbanismo, Ministerio de Desarrollo Social, Instituto Nacional de Estadísticas (INE), FAHNEU, Fundación mi Parque y Centro de Políticas Públicas UC (2018), Datos oficiales del MINVU (2013)

Kolmogorov, A. N., Combinatorial foundations of information theory and the calculus of probabilities, Russian Mathematical Surveys, 38, 4, 29-40 (1983)

Liu, Y., Ch. Liu y D. Wang, Understanding Atmospheric Behaviour in Terms of Entropy: A Review of Applications of the Second Law of Thermodynamics to Meteorology, doi: 10.3390/cl3010211, Entropy, 13, 211-240 (2011)

Lopez-Corona, O., P. Padilla y otros seis autores, Measuring social complexity and the emergence of cooperation from entropic principles, Physics-soc-ph. arXiv, 1502.05741v2[physics. Soc-ph] (2015)

Martínez, J. A. y F. A. Vinagre, La entropía de Kolmogorov, su sentido físico y su aplicación al estudio de lechos fluidizados 2D, Departamento de Química Analítica e Ingeniería Química, Universidad de Alcalá, Alcalá de Henares, Madrid, Academia (2016)

Mahulikar, S.P y H. Herwig, Exact thermodynamic principles for dynamic order existence and evolution in chaos, Chaos Soliton. Fractal, 41(4), 1939-1948 (2009)

Morán, M. J. y H. N. Shapiro, Fundamentos de Termodinámica Técnica, 2a Ed., Reverte, Barcelona, España (2005) 
Parker, D., The H - Theorem, Molecular Disorder and Probability: Perspectives from Boltzmann's Lectures on Gas Theory, PhilSci - Archive, OAI - Open Archives Initiative (2009)

Prigogine, I., Introduction to Thermodynamics of Irreversible Processes, $1^{\text {st }}$ Ed., Charles C. Thomas Publisher, Springfield, IL, USA (1955)

Ramos, Y., B. St-Onge, J.P. Blanchet y A. Smargiassi, Spatio-temporal models to estimate daily concentrations of fine particulate matter in Montreal: Kriging with external drift and inverse distance-weighted approaches, doi:10.1038/jes.2015.79, Journal of Exposure Science and Environmental Epidemiology, 26, 405-414 (2016)

Ruelle, D. P., Extending the definition of entropy to nonequilibrium steady states, Proc. Natl. Acad. Sci. USA, 100(6), 3054 -3058 (2003)

Sayre, N.F., The Genesis, History, and Limits of Carrying Capacity, doi: 10.1080/00045600701734356, Annals of the Assoc. of American Geo., 98(1), 122- 134 (2008).

Shannon, C., A mathematical theory of communication, doi:10.1002/j.1538-7305.1948.tb01338.x, Bell System Technical Journal, 27, 379-423 y 623-656 (1948)

Salini. G.A. y P. Pérez, A Study of the Dynamic Behaviour of Fine Particulate Matter in Santiago, Chile, doi: 10.4209/aaqr.2013.10.0323, Aerosol and Air Quality Research, 15, 154-165 (2015)

Shaw, R., Strange attractors, chaotic behavior, and information flow, Zeitschrift für Naturforschung A, 36, 80-112 (1981)

Sinai, Ya. G., On the concept of entropy of a dynamical system, Doklady Akademii nauk Souiza Sovetskikh Sotsialisticheskikh Respublik, 124, 768-771 (1959)

Sprott, J. C., Chaos and Time - Series Analysis, $4^{\text {th }}$ Ed., Oxford University Press, New York, USA (2006)

Wackernagel, M., N.B. Schulz y otros nueve autores, Tracking the ecological overshoot of the human economy, doi: 10.1073/pnas.142033699, Proc. Natl. Acad.Sci. U.S.A., 99 (14), 9266-9271 (2002)

Wolf, A., J. B. Swift, H.L. Swinney y J.A. Vastano, Determining Lyapunov exponents from a time series, Physica 16D, 285317, North-Holland, Amsterdam (1985) 
